Research Article

\title{
To Study the Prevalence of Osteopenia and Osteoporosis in Patients with COPD and to Correlate Severity of COPD with Prevalence of Osteopenia and Osteoporosis
}

\author{
Priyanka', Jayanti Khura' ${ }^{2}$, Shalini Shakarwal ${ }^{3}$, Pratap Singh ${ }^{4}$ \\ ${ }^{1,2}$ Senior Resident, ${ }^{4}$ Professor of Medicine, Department of Medicine, ABVIMS \& Dr RML Hospital, Delhi, India. \\ ${ }^{3}$ Assistant Professor, Department of Obstetrics \& Gynaecology, MAMC \& Lok Nayak Hospital, Delhi, India. \\ DOI: https://doi.org/10.24321/2349.7181.202106
}

\section{I $\quad \mathbf{N} \quad \mathbf{F} \quad \mathbf{O}$}

\section{Corresponding Author:}

Pratap Singh, Department of Medicine, ABVIMS

\& Dr RML Hospital, Delhi, India.

E-mail Id:

drpratapsingh@yahoo.co.in

Orcid Id:

https://orcid.org/0000-0001-6291-4287

How to cite this article:

Priyanka, Khura J, Shakarwal S, Singh P. To Study the Prevalence of Osteopenia and Osteoporosis in Patients with COPD and to Correlate Severity of COPD with Prevalence of Osteopenia and Osteoporosis. J Adv Res Med. 2021; 8(2): 1-4.

Date of Submission: 2021-06-05

Date of Acceptance: 2021-06-28

\section{$\begin{array}{llllllll}\mathbf{A} & \mathbf{B} & \mathbf{S} & \mathbf{T} & \mathbf{R} & \mathbf{A} & \mathbf{C} & \mathbf{T}\end{array}$}

Chronic Obstructive Pulmonary Disease (COPD) is a chronic respiratory airway disease characterized by both pulmonary and systemic inflammatory response and associated extra-pulmonary complications. Female gender, age and smoking are common pathogenic risk factors for both COPD and osteoporosis. Many other factors like low daily physical activity, chronic malnutrition, hypogonadism, vitamin D deficiency and chronic steroid therapy are risk factors for osteoporosis.

Objectives: The study was done to evaluate the prevalence of osteopenia and osteoporosis in patients of COPD and to correlate the severity of COPD with osteopenia/ osteoporosis.

Material \& Method: The study was conducted at a tertiary care hospital in Delhi after ethical clearance from institutional review board. Total of 76 patients of both genders were taken. COPD diagnosis and staging was done as per Global Initiative for Chronic Obstructive Lung Disease (GOLD) criteria. Bone mineral density (BMD) was evaluated by DEXA scan in all patients. All data was entered in preformed data sheets. Statistical analysis for association of COPD with osteoporosis was done by chi-square test.

Result: Mostly patients were having either osteopenia or osteoporosis, only $6 \%$ patients were having normal BMD. COPD stage III-IV were having significant bone mass loss. Those having long smoking history were more osteoporotic as compared to others.

Conclusion: There is a very high prevalence of osteopenia and osteoporosis in COPD patients especially those with prolonged smoking history, GOLD stage III-IV. 35.5\% of COPD patients had osteopenia and $57.9 \%$ had osteoporosis.

Keywords: COPD, Dual-Energy X-ray Absorptiometry (DEXA), Osteoporosis, Global Initiative for Chronic Obstructive Lung Disease (GOLD) 


\section{Introduction}

Chronic Obstructive Pulmonary Disease (COPD) is a disease of increasing public health importance around the world. According to Global Initiative for Chronic Obstructive Lung Disease (GOLD), COPD is a common preventable and treatable disease which is characterized by persistent airflow limitation that is usually progressive and associated with an enhanced chronic inflammatory response in the lung to noxious particles or gases. ${ }^{1}$ Recurrent exacerbations and other associated comorbidities contribute to the overall severity in individual patient. COPD remains a major public health problem and is projected to be rank fifth, in year 2024, in burden of disease worldwide. In India, it is estimated that there are around 1.49 crore chronic cases of COPD in the age group of 30 years and above. In literature, it is stated that in most Western world, while the peak incidence of osteoporosis occurs at about 70-80 years of age, in India it may afflict $10-20$ years younger age group as compared to the western world. In normal Indian population, $29.9 \%$ of women and $24.3 \%$ of men between the age group of 20 and 79 years have low bone mass, while about $50 \%$ women and $36 \%$ of men over 50 years of age have low bone mass as compared to the western population. Its prevalence is $5.0 \%$ amongst Indian males and approximately $3.2 \%$ among the Indian females over 35 years of age. ${ }^{2}$ Patients with moderate to severe COPD often have multi-organ disease like skeletal muscle wasting, lung cancer (small cell, non-small cell), pulmonary artery hypertension, ischemic heart disease, vascular endothelial dysfunction with peripheral vascular disease, obstructive sleep apnoea, depression and osteoporosis. ${ }^{3}$

Osteoporosis is defined as a skeletal disorder characterized by compromised bone strength, predisposing a person to an increased risk of fracture. The fracture risk depends on bone strength, which is determined by bone mineral density (BMD) and bone quality. ${ }^{4}$ However, due to the absence of clinical tools for precise evaluation of bone quality, a diagnosis of osteoporosis has been reliant on BMD measured by Dual Energy X-ray Absorptiometry (DEXA): osteoporosis is diagnosed when BMD is 2.5 standard deviations or more below the young adult mean (T-score is equal to or less than -2.5) according to the World Health Organization criteria. ${ }^{4}$ Evidence suggests that the prevalence of osteoporosis in patients with COPD is high and potentially important with disease progression. As the disease progresses, osteoporosis becomes a more prevalent finding in the patients of COPD. 5,6

Several studies have shown an extremely high prevalence of osteoporosis and low BMD in patients with COPD, even in milder stages of the disease. ${ }^{7}$ Over half of the patients with COPD recruited for the large TORCH (Towards a Revolution in COPD Health) trial (6,000 patients) had osteoporosis or osteopenia as determined by DEXA. ${ }^{8}$ In one study, the prevalence of osteoporosis was $75 \%$ in patients with GOLD stage IV disease and was strongly correlated with reduced Fat Free Mass (FFM)..$^{9,10}$ Osteoporosis is often disabling in these patients and may be equally disabling as COPD itself. However, osteoporosis often remains undiagnosed in these patients and may impair respiratory function even further, if the patient experiences vertebral compressions and loss of height. ${ }^{5,11}$

Osteoporosis-associated fractures may further deteriorate pulmonary function and impair activities of daily life of COPD patients. Thus, the two diseases will form a vicious cycle, causing significant burden on these patients.

\section{Material and Method}

The study was a hospital-based cross-sectional study and had been conducted by recruiting total of 76 patients including both genders, attending Out Patient Department (OPD) of Medicine and Chest clinic along with some indoor patients who were admitted in Medicine wards, at Dr RML Hospital, Delhi for a period of one year. All the patients aged $\leq 70$ years, who were diagnosed with COPD, according to GOLD criteria were included in the study.

The diagnosis of COPD was made on clinical history/ examination and Pulmonary Function Testing (PFT). The patients who fulfilled the exclusion criteria based on history and examination were excluded from the study. Exclusion criteria in our study were Cushing's disease, rheumatic diseases, diseases affecting bones like CKD, CLD, hyperparathyroidism, bronchogenic carcinoma, thyroid dysfunction and patients receiving bone forming drug. Informed consent was obtained from all the participants. All the data were recorded on a pre-designed proforma. After doing the routine investigations, all patients were subjected to Pulmonary Function Tests (PFT) by Spirometry as per standard protocol. After staging all the patients into various stages of COPD as per GOLD criteria, all patients were investigated by DEXA scan for BMD at lumbar and hip spine for finding out the osteopenia or osteoporosis in these patients. World Health Organization (WHO) criterion was taken for defining the osteoporosis and was applied to all patients with T-score of $>-$ - 2.5 standard deviation (SD) was diagnosed to have osteoporosis, -1 SD to -2.5 SD were diagnosed to have osteopenia and < - 1 SD as normal. ${ }^{5}$ In our study, BMD had been measured by 05 Prodigy ProBone Densitometry System, Wipro GE Healthcare machine.

Statistical analysis for association of COPD with osteoporosis was done by chi-square test.

\section{Result and Observation}

The present study was undertaken to study the prevalence of osteopenia and osteoporosis in patients of COPD and to correlate severity of COPD with prevalence of osteopenia 
and osteoporosis. In our study, we had observed that, out of the total 76 patients, majority of the patients of COPD had osteopenia/ osteoporosis. Only $6.6 \%(n=5)$ patients had normal BMD, 35.5\% $(n=25)$ had osteopenia, while $57.9 \%(n=46)$ had osteoporosis. Among males 32\% $(n=16)$ had osteopenia, while $62 \%(n=31)$ had osteoporosis and $6 \%(n=3)$ had normal BMD. Among females $42.3 \%(n=11)$ had osteopenia, while $50 \%(n=13)$ have osteoporosis and 7.7\% $(n=2)$ have normal BMD. Incidence of osteoporosis in menopausal women was $52.4 \%(n=15)$ and observed higher, as compared to pre-menopausal, $40 \%(n=11)$ women. The difference in mean age of normal BMD group (54.8 \pm 10.52 ) and osteoporosis $(57.17 \pm 7.90)$ was statistically significant $p<0.05$. Demographic profile of the patients and their BMD status is represented in Table 1 and BMD status in different stages of COPD is shown in Table 2.

Among smokers $(n=59)$, only $6.8 \%(n=4)$ had normal BMD, whereas $35.6 \%(n=21)$ had osteopenia and $57.6 \%(n=34)$ had osteoporosis. Among non-smokers ( $n=17)$, only $5.9 \%(n=1)$ had normal BMD, whereas $35.3 \%(n=6)$ had osteopenia and $58.8 \%(n=10)$ had osteoporosis. Patients who had smoking history of $>10$ yrs had statistically higher prevalence of osteoporosis compared to those who had smoked for $\leq$ 10 yrs (88.2\% vs $11.8 \%, \mathrm{p}<0.0001)$. Osteoporotic patients
( $n=34)$ with history of smoking had statistically higher mean pack years as compared to normal BMD smokers (29.47 \pm 10.66 Vs $5.0 \pm 2.94$ ).

Patients who had history of inhaled corticosteroid (ICS) use for $>2$ yrs had statistically higher prevalence of osteoporosis compared to those who had history of ICS use for $\leq 2 \mathrm{yrs}$ (100\% vs 48.3\%, $\mathrm{p}<0.0001)$.

Mean duration of COPD was significantly lower in normal BMD group (1.86 $\pm 1.07 \mathrm{yrs}$ ) as compared to osteoporosis group ( $8.43 \pm 1.66$ yrs) $p<0.05$. Osteopenia and osteoporosis were affected significantly by the duration of COPD. The prevalence of osteoporosis increased with severity of COPD (28.6\% in GOLD stage I, 38.2\% GOLD stage II, 78.3\% GOLD stage III and $91.7 \%$ GOLD stage IV (Figure 1).

Osteoporosis was found in majority of patients with COPD stage III and IV disease. Osteopenia and osteoporosis was more frequently noticed at lumbar spine than hip region (Figure 2). The patients having osteoporosis at hip area also had osteoporosis at lumbar area but the vice versa is not true. It was also observed significantly that those who smoked more than 10 years and frequently took steroids in oral or inhaled form had significant osteoporosis as shown in below study.

Table I.BMD Status and Demographic Profile of Patients

\begin{tabular}{|c|c|c|c|c|c|c|}
\hline \multirow{2}{*}{ Variable } & \multicolumn{2}{|c|}{ Normal $(n=5)$} & \multicolumn{2}{|c|}{ Osteopenia (n=27) } & \multicolumn{2}{|c|}{ Osteoporosis $(n=44)$} \\
\hline & Mean & SD & Mean & SD & Mean & SD \\
\hline Mean age (yrs) & 54.8 & 10.52 & 52.03 & 6.73 & 57.17 & 7.90 \\
\hline Female gender $(n=26)$ & \multicolumn{2}{|c|}{$2(7.7 \%)$} & \multicolumn{2}{|c|}{$11(42.3 \%)$} & \multicolumn{2}{|c|}{$13(50 \%)$} \\
\hline Male gender $(n=50)$ & \multicolumn{2}{|c|}{$3(6 \%)$} & \multicolumn{2}{|c|}{$16(32 \%)$} & \multicolumn{2}{|c|}{$31(62 \%)$} \\
\hline Mean duration of COPD (yrs) & \multicolumn{2}{|c|}{$1.86 \pm 1.07$} & \multicolumn{2}{|c|}{$2.20 \pm 1.04$} & \multicolumn{2}{|c|}{$8.43 \pm 1.66$} \\
\hline On ICS (n=66) & \multicolumn{2}{|c|}{$5(7.6 \%)$} & \multicolumn{2}{|c|}{$25(37.9 \%)$} & \multicolumn{2}{|c|}{$36(54.5 \%)$} \\
\hline Not on ICS $(n=10)$ & \multicolumn{2}{|c|}{0} & \multicolumn{2}{|c|}{$2(20 \%)$} & \multicolumn{2}{|c|}{$8(80 \%)$} \\
\hline History of ICS used $>24$ months $(n=8)$ & \multicolumn{2}{|c|}{0} & \multicolumn{2}{|c|}{0} & \multicolumn{2}{|c|}{$8(100 \%)$} \\
\hline Tobacco Smoking $(n=59)$ & \multicolumn{2}{|c|}{$4(6.8 \%)$} & \multicolumn{2}{|c|}{$21(35.6 \%)$} & \multicolumn{2}{|c|}{$34(57.6 \%)$} \\
\hline$>10 y r s$ of smoking $(n=31)$ & \multicolumn{2}{|c|}{0} & \multicolumn{2}{|c|}{$1(3.2 \%)$} & \multicolumn{2}{|c|}{$30(96.8 \%)$} \\
\hline Mean pack yrs of smoking & \multicolumn{2}{|c|}{$5.0 \pm 2.94$} & \multicolumn{2}{|c|}{$9.0 \pm 3.10$} & \multicolumn{2}{|c|}{$29.47 \pm 10.66$} \\
\hline Menopause $(n=21)$ & \multicolumn{2}{|c|}{$2(9.5 \%)$} & \multicolumn{2}{|c|}{$8(38.1 \%)$} & \multicolumn{2}{|c|}{11 (52.4\%) } \\
\hline
\end{tabular}

Table 2.Association between COPD Stages and Bone Mineral Density

\begin{tabular}{|c|c|c|c|c|c|c|c|c|}
\hline \multirow{3}{*}{ BMD } & \multicolumn{8}{|c|}{ COPD STAGES } \\
\hline & \multicolumn{2}{|c|}{ I (n=7) } & \multicolumn{2}{|c|}{ II $(n=34)$} & \multicolumn{2}{|c|}{ III $(n=23)$} & \multicolumn{2}{|c|}{ IV $(n=12)$} \\
\hline & No. $[\mathrm{M} / \mathrm{F}]$ & $\%$ & No. $[\mathrm{M} / \mathrm{F}]$ & $\%$ & No. [M/F] & $\%$ & No. $[\mathrm{M} / \mathrm{F}]$ & $\%$ \\
\hline Normal $(n=5)$ & $2[1 / 1]$ & 28.6 & $3[2 / 1]$ & 8.9 & 0 & 0.0 & 0 & 0.0 \\
\hline Osteopenia (n=27) & $3[2 / 1]$ & 42.9 & $18[12 / 6]$ & 52.9 & $5[2 / 3]$ & 21.7 & $1[0 / 1]$ & 8.3 \\
\hline Osteoporosis $(n=44)$ & $2[1 / 1]$ & 28.6 & $13[9 / 4]$ & 38.2 & $18[12 / 6]$ & 78.3 & $11[9 / 2]$ & 91.7 \\
\hline
\end{tabular}




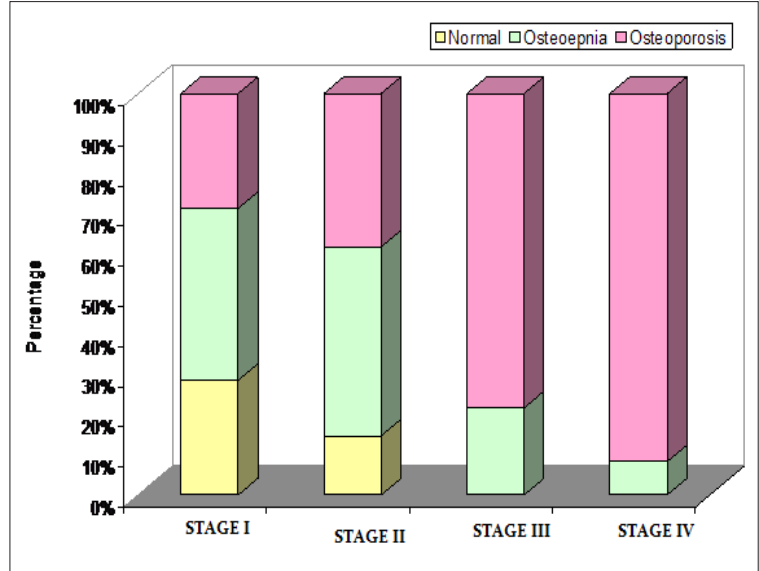

Figure I.Relation of COPD Staging and BMD Results

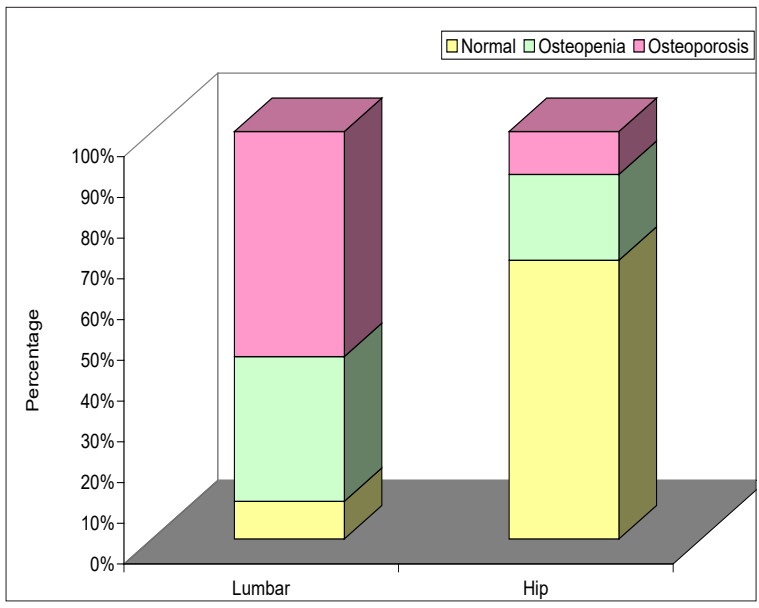

Figure 2.Distribution of Osteoporosis According to Site

\section{Discussion}

COPD is a chronic inflammatory airway and parenchymal disease, associated with various systemic comorbidities including osteoporosis. Osteoporosis and its related fractures are much common and have significant impacts on quality of life. DEXA along with other biochemical bone turnover markers are also useful for noninvasively evaluate bone metabolism..$^{12}$ Increase in bone markers, example, high bone turnover, has been shown to be not only a predictor of future BMD decline but also a BMD-independent risk factor of fracture in primary osteoporosis. It has been observed that COPD-associated osteoporosis is extremely under diagnosed as well as under-treated. Study done by Daga et al., also showed that osteoporosis is more prevalent among COPD patients than healthy subjects. ${ }^{13}$ Thus, it is also important to recognize other risk factors and strategies to manage osteoporosis in COPD patients to avoid osteoporotic fractures that deteriorate quality of life and future prognosis of the disease. The present study also supports the increased prevalence of osteoporosis in COPD patients. Also, various risk factors like chronic inhaled steroids, prolonged oral steroid therapy, poor nutrition and recurrent exacerbation of COPD are also associated with reduced $B M D$ in patients with COPD.

There are only a limited number of studies investigating bone turnover in COPD patients. Some of the recent studies ${ }^{14}$ have suggested that both decreased bone mineral density (BMD) and impaired bone quality contribute to bone fragility, causing fractures in COPD patients. Slemenda et al. ${ }^{15}$ reported that lumbar spine BMD was $12 \%$ lower in smokers who have smoked 20 pack years compared to non-smokers. It has been observed that prolonged smoking history has been shown to be an independent risk factor for osteoporosis both in men and women as shown in our study also. Patients with COPD who are often being treated with inhaled, oral, parenteral glucocorticoids, during exacerbations and such glucocorticoid therapy along with various other risk factors clearly increase the risk for the development and progression of osteoporosis in COPD patients. These finding also had been reported in study done Daga et al., which showed prevalence of osteoporosis in COPD in the range of 9-69\% ${ }^{16}$ Osteoporosis related fractures are associated with several adverse health outcomes in COPD, including an increase hospitalization along with loss of Disability-Adjusted Life-Years (DALYs) and increased mortality rates. ${ }^{17}$ Similar finding have been observed in our study also.

It has also been observed that after complete cessation of smoking, there is increase in $\mathrm{BMD}$ along with improvement in quality of life if complete abstinence is being maintained for smoking. Hence, a routine workup to screen all COPD patients for osteopenia/ osteoporosis should be done by DEXA. Early diagnosis and timely treatment of osteoporosis will prevent morbidity and significant loss of DALYs because of bone fractures. Also, all COPD patients should be counselled and followed on regular basis for complete cessation of smoking. It would further prevent other extra-pulmonary comorbidities and complications in COPD patients.

\section{Conclusion}

Our study showed that there is a high prevalence of osteopenia and osteoporosis in COPD patients especially those with prolonged smoking history, GOLD stage III-IV. Also it has been observed that lumbar spine is much more affected than hip region in COPD patients in our study.

\section{Conflict of Interest: None}

\section{References}

1. Pauwels RA, Buist AS, Calverley PM, Jenkins CR, Hurd SS Global strategy for the diagnosis, management, and prevention of chronic obstructive pulmonary disease. NHLBI/WHO Global Initiative for Chronic Obstructive Lung Disease (GOLD) Workshop summary. Am J Respir Crit Care Med. 2001;163(5):1256-76. [PubMed] [Google 
Scholar]

2. Jindal SK. COPD: The unrecognized epidemic in India. J Asso Physicians India. 2012;60:14-16. [PubMed] [Google Scholar]

3. Barnes PJ and Celli BR. Systemic manifestations, and comorbidities of COPD. Eur Respair J .2009; 33: 11651185. [PubMed] [Google Scholar]

4. Daisuke Inoue, Reiko Watanabe, Ryo Okazaki. COPD and osteoporosis: links, risks, and treatment challenges. Int J Chron Obstruct Pulmon Dis. 2016;11:637-648. [PubMed] [Google Scholar]

5. Leech JA, Dulberg C, Kellie S, Pattee L, Gay J. Relationship of lung function to severity of osteoporosis in women. Am Rev Respir Dis. 1990;141:68-71. [PubMed] [Google Scholar]

6. Walsh LJ, Wong CA, Oborne J, Cooper S, Lewis SA, Pringle M, Hubbard R, Taatersfield AE. Adverse effects of oral corticosteroids in relation to dose in patients with lung disease. Thorax. 2001;56:279-284. [PubMed] [Google Scholar]

7. Jorgensen NR, Schwarz P. Osteoporosis in chronic obstructive pulmonary disease patients. Curr Opin Pulm Med 2008; 14:122-127. [Google Scholar]

8. The TORCH Study Group. The TORCH (Towards a Revolution in COPD Health) survival study protocol. Eur Respir J. 2004; 24:206-10. [PubMed] [Google Scholar]

9. Vrieze A, de Greef MH, Wijkstra PJ, Wempe JB. Low bone mineral density in COPD patients related to worse lung function, low weight and decreased fat-free mass. Osteoporosis Int 2007; 18: 1197-1202. [PubMed] [Google Scholar]

10. Jorgensen NR, Schwarz P, Holme I, Henriksen BM, Petersen $\mathrm{L}$, Backer V. The prevalence of osteoporosis in patients with chronic obstructive pulmonary disease: a cross sectional study. Respir Med 2007; 101: 177-185. [PubMed] [Google Scholar]

11. Biskobing DM. COPD and osteoporosis. Chest. 2002; 121:609-620. [PubMed] [Google Scholar]

12. Naylor K, Eastell R. Bone turnover markers: use in osteoporosis. Nat Rev Rheumatol. 2012;8(7):379-389. [PubMed] [Google Scholar]

13. Daga MK, Bharali D, Gupta B, Mawari G, Hira HS. Assessment of osteoporosis and osteopenia and its co-relation with disease severity in patients of chronic obstructive pulmonary disease with respect to associated risk factors: A case control study in India. J Pulm Respir Med. 2018;8:456-61. [Google Scholar]

14. Incalzi RA, Caradonna P, Ranieri P, Basso S, Fuso L, Pagano F, Ciappi G, Pistelli R. Correlates of osteoporosis in chronic obstructive pulmonary disease. Respir Med. 2000; 94: 1079-84.

15. Slemenda CW, Hui SL, Longcope $C$, et al. Cigarette smoking, obesity, and bone mass. J Bone Miner Res
1989;4:737-741

16. Daga MK, Kumar N, Raghav RV, Marwari G, Chand S, Aarthi J, Jha MK, Hussain M. Osteoporosis in chronic obstructive pulmonary disease: More than just a comorbidity. J Adv Res Med. 2020; 3:7-12. [Google Scholar]

17. Gan WQ, Man SFP, Senthilselvan A, Sin DD . Association between chronic obstructive pulmonary disease and systemic inflammation: a systematic review and a meta-analysis. Thorax. 2004;59:574e80. [PubMed] [Google Scholar] 